\title{
Descripción de los resultados de las pruebas neuropsicológicas en el diagnóstico diferencial de los pacientes con Alzheimer*
}

\section{Neuropsychological Test Results Description in the Differential Diagnosis of Patients with Alzheimer's Disease \\ Descrição dos resultados dos testes neuropsicológicos no diagnóstico diferencial de pacientes com Alzheimer}

\author{
Maria Mercedes Hackspiel $Z^{\mathrm{a}}$ \\ Universidad Militar Nueva Granada, Colombia \\ maria.hakspiel@unimilitar.edu.co \\ ORCID: https://orcid.org/0000-0002-8191-6816 \\ Olga Paredes Garavito \\ Universidad Militar Nueva Granada, Colombia \\ ORCID: https://orcid.org/0000-0001-8303-0683
}

Recibido: 19 Diciembre 2019

DOI: https://doi.org/10.11144/Javeriana.ie22.drpn

\section{Resumen:}

Introducción: el deterioro cognitivo en las demencias es una de las dimensiones que requieren ser evaluadas de forma eficaz en los pacientes que acuden a los servicios especializados. Por décadas el Mini Mental (MMSE) y la evaluación cognitiva Monterreal (MoCA) se han constituido en los instrumentos más utilizados, sin embargo, la complejidad del diagnóstico ha dado origen a nuevas pruebas como la batería de evaluación frontal (FAB). Objetivo: describir los resultados de las pruebas neuropsicológicas aplicadas a los pacientes con demencias. Método: diseño: Estudio descriptivo transversal con 19 pacientes con demencias de diversas índoles, quienes diligenciaron pruebas psicológicas de tamizaje. El encuadre fue el de la bioética, especialmente con la visión de la dignidad y autonomía de los pacientes. Resultados: los puntajes evidencian deterioro cognitivo moderado y la presencia del diagnóstico de Alzheimer, adicionalmente, el FAB evidencia cortes para diagnóstico de Alzheimer. Conclusiones: a pesar de que cada instrumento evidencia puntos de corte más sensibles a cada tipo de demencia, las dos pruebas evidencian similitudes en cuanto a curva de puntuación en cada criterio de evaluación. Es importante que los pacientes con deterioro cognitivo tengan acceso a pruebas que permitan realizar un diagnóstico diferencial adecuado pues de este dependerá no solo la intervención y tratamiento sino el cuidado que recibirá en el proceso de la enfermedad.

Palabras clave: Disfunción cognitiva, diagnóstico diferencial, pruebas neuropsicológicas, enfermedad de Alzheimer, demencia.

\section{Abstract:}

Introduction: cognitive impairment in dementia is one of the dimensions that needs to be evaluated effectively in patients who go to specialized services. For decades, the Mini Mental State Exam (MMSE) and the Monterreal cognitive assessment (MoCA) have been the most widely used instruments; however, the complexity of the diagnosis process has given rise to new tests such as the frontal assessment battery (FAB). Objective: to describe the results of neuropsychological tests applied to patients with dementia. Method: design: a descriptive, cross-sectional study of 19 patients with various types of dementia, who completed psychological screening tests. The context was that of bioethics, especially with a vision towards the dignity and autonomy of the patients. Results: the scores show moderate cognitive deterioration and the presence of a diagnosis of Alzheimer's disease; additionally, the FAB shows cut-off points for the diagnosis of Alzheimer's disease. Conclusions: despite the fact that each instrument shows cut-off points that are more sensitive to each type of dementia, both tests show similarities in terms of the scoring curve for each evaluation criterion. It is important for patients with cognitive impairment to have access to tests that allow an adequate differential diagnosis to be performed, since not only the intervention and treatment will depend on this but also the care that they will receive during the course of the disease.

Keywords: Cognitive dysfunction, differential diagnosis, neuropsychological tests, Alzheimer's disease, dementia.

\section{Resumo:}

Introdução: o comprometimento cognitivo nas demências é uma das dimensões que precisam ser avaliadas de forma eficaz em pacientes que recorrem a serviços especializados. Por décadas, o Mini Mental (MMSE) e a avaliação cognitiva Monterreal (MoCA) tornaram-se os instrumentos mais utilizados, porém, a complexidade do diagnóstico deu origem a novos testes como a bateria de avaliação frontal (FAB). Objetivo: descrever os resultados dos testes neuropsicológicos aplicados a pacientes com

Notas de autor

\footnotetext{
a Autora de correspondencia. Correo electrónico: maria.hakspiel@unimilitar.edu.co
} 
demências. Método: desenho: Estudo descritivo transversal com 19 pacientes com demências de diversos tipos, quem realizaram testes psicológicos de triagem. O enquadre foi o de bioética, especialmente com o olhar da dignidade e autonomia dos pacientes. Resultados: os escores evidenciam deterioração cognitiva moderada e a presencia do diagnóstico de Alzheimer. Além disso, o FAB evidencia cortes para diagnóstico de Alzheimer. Conclusões: apesar de cada instrumento evidenciar pontos de corte mais sensíveis a cada tipo de demência, os dois testes evidenciam semelhanças quanto à curva de pontuação em cada critério de avaliação. É importante que os pacientes com comprometimento cognitivo tenham acesso a testes que permitam um diagnóstico diferencial adequado pois dele dependera não só a intervenção e o tratamento mas também os cuidados que receberá no processo de adoecimento.

Palavras-chave: s: Disfunção cognitiva, diagnóstico diferencial, testes neuropsicológicos, doença de Alzheimer, demência.

\section{Introducción}

El paciente con deterioro cognitivo requiere de una valoración seria, que defina, por un lado, los aspectos inherentes de un posible declive de la función fisiológica, como en el caso del envejecimiento y, por otro lado, la manera en que el deterioro se manifiesta, aportando a un diagnóstico más claro, indistinto de la edad, pero si resultante de la condición médica que lo compaña (por demencia, síndrome de Down, etc.).

Si bien la valoración debe ser integral, es decir sobre indicadores físicos, funcionales, psicológicos, sociales, etc. Las pruebas para la estimación del estado cognitivo ayudan además a definir la reversibilidad o progresión de esta y también la etapa de la enfermedad, lo que permite un mejor diagnóstico y pronóstico de cada paciente. En el caso de enfermedades como las demencias, la característica son los varios déficits cognoscitivos, sumados al deterioro de la memoria y adicional a otras alteraciones como la afasia o la agnosia, y también, como lo plantean Peralta y cols (1), cambios atípicos en la histología cerebral que lo diferencian de un envejecimiento benigno, es decir, típicos de la senectud.

Entre las pruebas más utilizadas para la detección y valoración de demencias, se encuentran las baterías neuropsicologías, con subtest de funciones ejecutivas como el examen cognoscitivo Mini Prueba del Estado Mental o Mini Mental State Examination de Folstein (2), conocido por sus siglas en inglés MMSE. Esta escala psicométrica breve, busca la detección misma de demencia en pacientes geriátricos mayoritariamente, e indaga el "estado mental" que permite de acuerdo con sus autores, diferenciar los trastornos funcionales orgánicos.

Otra de las pruebas más utilizadas es la Evaluación Cognitiva Motereal, más llamada por sus siglas, MoCA en 1996, que se convirtió en un instrumento de tamizaje de deterioro cognitivo leve. Al igual que el MMSE, evalúa diferentes dominios cognitivos como la atención y concentración, funciones ejecutivas, memoria, lenguaje, habilidades viso constructivas, pensamiento conceptual, cálculo y orientación (3).

Ahora bien, existen resultados variados respecto a sus ventajas, por ejemplo, Fiorenzato et al. (4), aseguran que MoCA es un instrumento más sensitivo al detectar deficiencia cognitiva que otras pruebas, sin embargo, su grupo poblacional de estudio se concentró en pacientes con Parkinson y atrofia sistémica múltiple, por lo que ellos mismos sugieren incluir otro tipo de patologías y pruebas de detección. Por su parte, Dong et al. (5), aseguran que el MoCA también es superior que el MMSE para la detección de demencia y daño de tipo vascular, así como quienes lo eligen sobre el Mini Mental para una detección más fiable de deterioro cognitivo temprano (6).

Sin embargo, cuando la población cambia a personas con demencias tipo Alzheimer, especialmente en estadios más avanzados, los resultados de los estudios también arrojan evidencias más complejas. Uno de estos estudios evaluó los factores relacionados con el deterioro cognitivo moderado en la enfermedad de Alzheimer (EA); se utilizó los instrumentos MoCA y MMSE y sus resultados se compararon con información clínica relevante. Durante el seguimiento de año y medio, se concluye que quienes obtuvieron puntajes de deterioro moderado en las dos pruebas progresaron a enfermedad de Alzheimer; la hiperlipidemia e Índice de masa corporal mayor a $24 \mathrm{~kg} / \mathrm{m}^{2}$, predijeron el deterioro de funciones cognitivas (7). 
También llama la atención los estudios de Benedictus et al. (8) quienes observaron el corte de puntaje en el Mini Mental para relacionarlo con el flujo cerebral. Si el flujo cerebral era menor al promedio y su puntaje en el MMSE era de 22, los pacientes con Alzheimer demostraban un deterioro cognitivo mucho más rápido, comparado con otros pacientes. Así pues, se puede concluir que no todos los subtest del Mini mental y mucho menos del MoCA son un identificador fundamental del deterioro cognitivo en la EA (9).

Al tornarse más complicadas y con necesidad de mayor especificación clínica y diagnóstica, las valoraciones neuropsicológicas para detección de Alzheimer traen al escenario una batería llamada Frontal Assesment Battery o FAB (10).

Delgado y Salinas $(11,12)$ comparan las 3 pruebas mencionadas para evaluar EA, estableciendo que, todas son útiles sobre todo para diagnósticos diferenciales. El MoCA lo consideraron más sensible que el MMSE para detección de deterioro vascular; el Mini Mental es poco sensible en deterioro de predominio disejecutivo y el FAB es útil en el diagnóstico diferencial entre EA y demencia frontotemporal y entre síndromes parkinsonianos.

Aunque los puntajes del MoCA y del Mini mental han sido "equiparados" en distintos estudios, no sucede lo mismo con entre el FAB y estas dos, a causa de sus subpruebas y los indicadores a medir (Tabla 1).

El diagnóstico médico siempre traerá consigo no solo la elección de la intervención terapéutica, sino una "identidad" distinta y unas prácticas de cuidado que serán la respuesta acorde a los resultados derivados de un diagnóstico. La gestión del cuidado se organiza acorde a unos resultados e incluso a un pronóstico derivado de estos. Las decisiones de la gestión del cuidado afectan el quehacer de los profesionales de salud sobre cómo deben cuidar y atender a esos pacientes. Es por esto que surgió el interés por profundizar en los indicadores de los resultados de las pruebas aplicadas a pacientes con demencias en el Servicio de Neurología y el efecto que estos podrían tener en el ejercicio y gestión del cuidado.

\section{Objetivo}

Describir los resultados de las pruebas neuropsicológicas aplicadas a los pacientes con demencias.

\section{Método}

Se realizó un estudio de tipo descriptivo que permitiera reseñar la frecuencia y las características más importantes de los resultados de pruebas neuropsicológicas aplicadas. La recogida de los datos se llevó a cabo durante tres meses en el servicio de Neurología de un Hospital de tercer nivel en Bogotá.

Se eligió un muestreo por conveniencia, compuesto por 19 pacientes que accedieron a participar de la lista conformada por el servicio de Neurología como voluntarios. Se incluyeron a los pacientes que se encontraban en espera de las pruebas neuropsicológicas, con diagnóstico de demencia en diferentes etapas y años de diagnóstico y que no contaran con mayores complicaciones para ser remitidos a una resonancia magnética funcional (necesaria para las otras etapas de la investigación). Se excluyeron a los pacientes que ya habían realizado las pruebas neuropsicológicas 6 meses antes, con diagnósticos interrogados de demencia o en últimas etapas de estas, que no pudieran sostener una entrevista en el consultorio y quienes presentar dificultades para ser sometidos a resonancia magnética por condiciones médicas. Dado que la muestra reclutada para las pruebas neuropsicológicas sería la misma que para la prueba adicional con resonancia magnética funcional, se tomó en cuenta varios comentarios como los de Reyes (13) donde se sostiene que un número necesario de sujetos que deben participar en un experimento es de 12 ampliándose a 25. Sin embargo, cumplir con todos los criterios de inclusión en el servicio de neurología en el momento dado, únicamente permitió 19 pacientes en la muestra final. 
Se aplicó la batería neuropsicológica escogida específicamente para esta fase de la investigación fueron las pruebas de Mini Mental y Frontal Assesment Battery (14). Estas se complementaron con el cuestionario sobre funcionalidad y sociodemográfico (autoría propia, que fue ya utilizado en investigaciones previas con pacientes con enfermedad de Alzheimer; tuvo un pilotaje de 12 pacientes en el año 2015 en una Fundación de orientación a la población).

Las puntuaciones se dieron de acuerdo con criterios de edad y escolaridad (de acuerdo con el protocolo de cada instrumento) y se contrastaron con el diagnóstico inicial dado según su historia clínica (Tabla 1).

TABLA 1.

Descripción de las pruebas de tamizaje MMSE, MoCA y FAB

\begin{tabular}{|c|c|c|}
\hline Test & (MMSE) & FAB \\
\hline Puntaje Máximo & $\begin{array}{l}\text { Puntaje máximo de } 30 \\
\text { puntos }\end{array}$ & Puntuación máxima 18 puntos \\
\hline Áreas evaluadas & $\begin{array}{l}\text { orientación, memoria } \\
\text { inmediata, atención y } \\
\text { cálculo, recuerdo } \\
\text { diferido, y lenguaje y } \\
\text { construcción }\end{array}$ & $\begin{array}{l}\text { Conceptualización (abstracción), } \\
\text { fluencia lexical (flexibilidad mental), } \\
\text { programación motora, sensibilidad a } \\
\text { las interferencias (tendencia a la } \\
\text { distracción), control inhibitorio y } \\
\text { autonomia. }\end{array}$ \\
\hline \multirow[t]{7}{*}{ Punto de corte } & $\begin{array}{l}\text { Puntaje arriba de } \\
24 \quad(26 \text { a nivel } \\
\text { internacional }) \text { o más es } \\
\text { considerado }\end{array}$ & $\begin{array}{l}\text { Puntuación de } 12 \text { es el punto de corte } \\
\text { para distinguir entre la demencia } \\
\text { frontal tipo ejecutivo y la demencia tipo } \\
\text { Alzheimer. }\end{array}$ \\
\hline & Punto de corte es 21 & $\begin{array}{l}\text { Ofrece dos puntos de corte para déficit } \\
\text { fronto-subcortical (15 sobre } 18) \text { y para } \\
\text { demencia fronto-subcortical (12 sobre } \\
\text { 18) }\end{array}$ \\
\hline & $\begin{array}{l}\text { Entre } 30 \text { y } 27: \text { Sin } \\
\text { Deterioro. }\end{array}$ & \\
\hline & $\begin{array}{l}\text { Entre 26 y 25: Dudoso o } \\
\text { Posible Deterioro. }\end{array}$ & \\
\hline & $\begin{array}{lcr}\text { Entre } 24 & \text { y } & 10: \\
\text { Demencia } & \text { Leve } & \text { a } \\
\text { Moderada. } & & \end{array}$ & \\
\hline & $\begin{array}{l}\text { Entre } 9 \text { y 6: Demencia } \\
\text { Moderada a Severa. }\end{array}$ & \\
\hline & $\begin{array}{l}\text { Menos de 6: Demencia } \\
\text { Severa. }\end{array}$ & \\
\hline \multirow[t]{5}{*}{$\begin{array}{l}\text { Aspectos } \\
\text { relevantes de la } \\
\text { calificación }\end{array}$} & $\begin{array}{l}\text { Es necesario diferenciar } \\
\text { entre pacientes de edad } \\
\text { superior a } 65 \text { años y los } \\
\text { de edad }\end{array}$ & I Sin restricción \\
\hline & igual o inferior a 65 . & \\
\hline & $\begin{array}{l}\text { - Pacientes }>65 \text { años, } \\
\text { puntuación inferior a } 23 \\
\text { es considerado } \\
\text { anormal. }\end{array}$ & \\
\hline & $\begin{array}{l}\text { - Pacientes no } \\
\text { geriátricos, puntuación } \\
\text { inferior a } 27 \text { es } \\
\text { considerado anormal. }\end{array}$ & \\
\hline & Educación en grados & \\
\hline
\end{tabular}

Aspectos éticos: Los participantes decidieron su participación voluntaria mediante el consentimiento informado (CI) para familiares y asentimiento escrito y verbal para los pacientes. El diligenciamiento consistió en la rigurosidad de aplicación bajo un contexto semejante al de un diálogo cotidiano, que 
permitiera generar un espacio de comprensión y siguiendo lo establecido en la Resolución 8430 de 1993. En la investigación, se resguardó el respeto por la dignidad y protección de los derechos y bienestar de los pacientes y su familia, aportando al conocimiento de los vínculos entre las causas de enfermedad, la práctica médica y la estructura social. El cuidado y la atención relativos a los riesgos se tuvieron presentes a cada momento, intensificándose en el caso de que la investigación revele contenidos protegidos o traumas colectivos, familiares o personales que comprometan la vida de los participantes (15).

\section{Análisis de datos}

Se llevó a cabo un análisis descriptivo de los datos, procesados a través del programa estadístico SPSS 25 (IBM). se usó la media como medida de tendencia central y se obtuvieron los porcentajes de prueba cumplida de acuerdo con la puntuación individual obtenida y el punto de corte.

\section{Resultados}

Los resultados de las pruebas neuropsicológicas son evidenciados respecto a algunos criterios del cuestionario sociodemográfico y del diagnóstico, sin embargo, no se tuvieron en cuenta para el presente artículo, las correlaciones con otras variables como sexo, edad, uso de medicamentos ni comorbilidad.

En el siguiente cuadro pueden observarse los datos obtenidos después de la aplicación de instrumentos (Tabla 2).

TABLA 2.

Puntuación del MMSE y FAB, diagnóstico inicial, tiempo con el diagnóstico

\begin{tabular}{|c|c|c|c|c|c|c|}
\hline Paciente & Diagnuóstico inicial & $\begin{array}{c}\text { Tiempo con el } \\
\text { diagnóstico }\end{array}$ & $\begin{array}{l}\text { Puntaje } \\
\text { MMSE }\end{array}$ & $\begin{array}{l}\text { Porcentaje } \\
\text { de prueba } \\
\text { aprobada }\end{array}$ & Puntaje FAB & $\begin{array}{l}\text { Porcentaje } \\
\text { de prueba } \\
\text { aprobada }\end{array}$ \\
\hline 1 & Sin confimar diagnóstico & 4-5 años & 24 & $80 \%$ & 7 & $83,80 \%$ \\
\hline 2 & Demencia & 3 años & 24 & $80 \%$ & 15 & $83,30 \%$ \\
\hline 3 & EA & 5 años & 15 & $50 \%$ & 3 & $38,80 \%$ \\
\hline 4 & Sin confimar diagnostico & 2 años & 19 & $63,30 \%$ & 10 & $55,50 \%$ \\
\hline 5 & EA & 10 años & 21 & $70 \%$ & 16 & $88,80 \%$ \\
\hline 6 & Demencia & 7 años & $\mathrm{NA}$ & $\mathrm{NA}$ & $\mathrm{NA}$ & $\mathrm{NA}$ \\
\hline 7 & EA & 2 años & 16 & $53,30 \%$ & 7 & $38,80 \%$ \\
\hline 8 & $\mathrm{EA}$ & 2 años & 3 & $10 \%$ & 4 & $22,20 \%$ \\
\hline 9 & $\mathrm{EA}$ & 1 año & 15 & $50 \%$ & 7 & $38,80 \%$ \\
\hline 10 & $\mathrm{EA}$ & 4 años & 6 & $20 \%$ & 5 & $27.70 \%$ \\
\hline 11 & EA & 10 años & 20 & $66,60 \%$ & 9 & $50 \%$ \\
\hline 12 & $\mathrm{EA}$ & 2 años & 27 & $90 \%$ & 8 & $44,40 \%$ \\
\hline 13 & Demencia vascular & 5 años (crisis de dos dias) & 24 & $80 \%$ & 9 & $50 \%$ \\
\hline 14 & EA & 4 años & 16 & $53,30 \%$ & 7 & $38,80 \%$ \\
\hline 15 & Demencia & 3 años & 28 & $93,30 \%$ & 14 & $77,70 \%$ \\
\hline 16 & Sin confimar diagnóstico & 10 años & 13 & $43,30 \%$ & 2 & $11,10 \%$ \\
\hline 17 & $\mathrm{EA}$ & 7 aũos & $\mathrm{NA}$ & $\mathrm{NA}$ & $\mathrm{NA}$ & $\mathrm{NA}$ \\
\hline 18 & EA & 2 años & 18 & $60 \%$ & 8 & $44,40 \%$ \\
\hline 19 & EA & 4 años & 23 & $76,60 \%$ & 11 & $61,10 \%$ \\
\hline Media & EA & 4,6 มños & 18 & $61 \%$ & 8 & $48,21 \%$ \\
\hline
\end{tabular}

La mayoría de los pacientes (once) del grupo tienen un diagnóstico de enfermedad de Alzheimer, los demás contaron con diagnóstico de demencia, sin especificar tipo, y uno con demencia vascular. Pocos de ellos (tres) no tienen aún un diagnóstico claro. El promedio de tiempo con el diagnóstico fue de 4.5 años; solo una paciente describe una recaída, en crisis, hacía dos días.

Los resultados obtenidos con el Mini mental tuvieron un promedio de 18 puntos y los del Frontal Assesment Battery de 8. Dos de los pacientes se encontraron en estados avanzados de deterioro al momento del diligenciamiento, por lo cual no pudieron completar la batería. 
De los once pacientes que tenían diagnóstico de enfermedad de Alzheimer, solo uno de ellos no tiene el punto de corte para el diagnóstico de esta enfermedad, de acuerdo con el FAB, aunque su puntuación corresponde a deterioro moderado en el MMSE.

Los resultados de dos pacientes que obtuvieron puntajes en el Mini mental muy bajos (3 y 6, correspondientes a demencia severa) y los puntajes más bajos en el FAB (4 y 5 para Alzheimer), siendo coherente su diagnóstico, con el daño cognitivo reflejado en la batería. Los demás pacientes con EA obtuvieron un promedio de 18 puntos en su desempeño cognitivo (MMSE) es decir, moderado y uno de 8 puntos en el FAB que evidencia presencia de EA.

Si bien es importante aclarar que las dos pruebas utilizadas no pueden ser equiparables dado su fundamento y porque, cada condición médica genera deterioro en regiones cerebrales distintas, pueden obtenerse impresiones reflejadas por la puntuación en los pacientes. Sin embargo, para un panorama general se presenta la Figura 1.

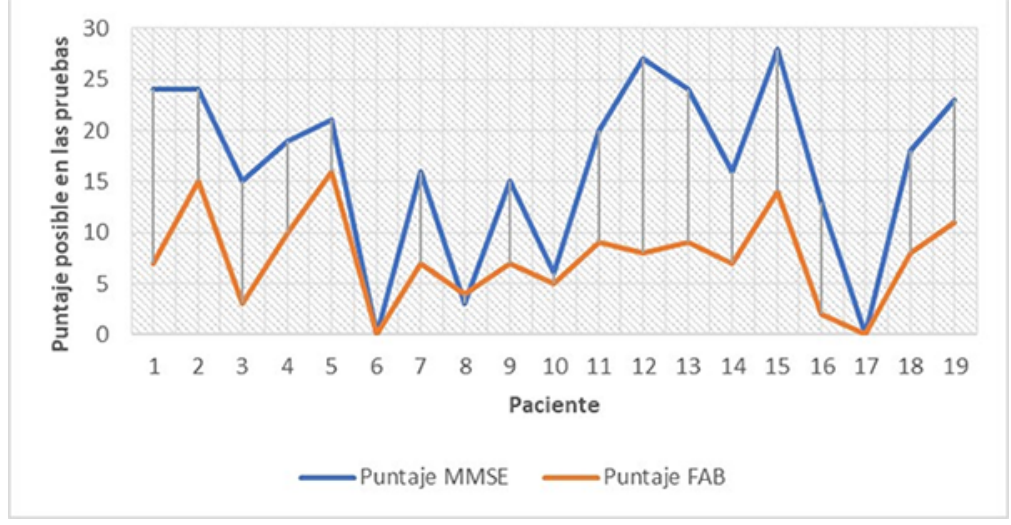

FIGURA 1.

Puntaje máximo alcanzado por cada paciente en las dos pruebas neuropsicológicas. Fuente: elaboración propia.

\section{Discusión}

De los pacientes con EA, uno de ellos tuvo punto de corte para el diagnóstico (de acuerdo con el FAB), este caso es de gran interés, dado su tiempo de diagnóstico que supera los 10 años pero que, a pesar de esto, no demuestra una pérdida notoria de ejecución a pesar de lo prolongado. Para autores como De la Vega y Zambrano (16) pacientes que obtienen entre 24 y 28 puntos en el Mini mental, a menudo pueden tener mal desempeño en las dimensiones relativas a la memoria, dado que algunos tipos de demencias presentan mayor déficit a este nivel. Al realizar un seguimiento longitudinal de estos pacientes, aproximadamente un $15 \%$ de ellos, evoluciona cada año, a la enfermedad de Alzheimer. Este aspecto podría evidenciarse con uno de los once pacientes, que evidencia los cortes límites tanto en el MMSE (23/24-DC moderado) y también para el FAB (11/12), pero no cuentan con un historial clínico previo que dé cuenta de la evolución del deterioro cognitivo anterior al momento de la aplicación.

Kim et al. (17) demuestran en su estudio, cómo el uso del FAB junto con el MSSE mejora el escaneo de la demencia, de todo tipo, incluyendo Alzheimer, a diferencia de solo el uso del mini mental. En términos generales, quienes obtuvieron puntajes más bajos al punto de corte en el FAB, también tienen puntuaciones de déficit moderado a severo en el MMSE.

Fueron "coincidentes" los resultados de los pacientes que obtuvieron puntajes en el Mini mental muy bajos (correspondientes a demencia severa) y los puntajes más bajos en el FAB (4 y 5 para Alzheimer), siendo coherente su diagnóstico, con el daño cognitivo reflejado en la batería. Estos dos pacientes, sin embargo, cuentan con pocos años de diagnóstico ( 2 y 4 años respectivamente); esto no necesariamente define su estatus 
de diagnóstico, pues en ocasiones los pacientes obtienen un diagnóstico formal tan solo años después de estar siendo tratado médicamente. Los demás con un promedio de 18 puntos (es decir, moderado) y uno de 8 puntos en el FAB que evidencia presencia de EA. Puntuación que podría explicarse como la evidencia de un daño en el desempeño de las funciones que se presentan ya en el Alzheimer y que se definen por su estadio, aspecto que no se pudo evaluar en la investigación por falta de información por parte de los familiares. Ferrer et al. (18), plantea en su estudio de correlación entre el FAB y diversas escalas de valoración funcional y neurológica que: las funciones evaluadas por la batería frontal no correlacionan con el promedio de deterioro funcional en las otras pruebas (DAD, TIN and FAQ) sin embargo, el FAB se correlaciona bien, con la intensidad del deterioro (medido con la GDS) y un test cognitivo breve (MMSE).

Las dificultades de las alteraciones cognitivas son bastante comunes, en muchas ocasiones por su comorbilidad o porque existe un dominio más afectado que otro y cobra mayor importancia.

Los diagnósticos son importantes para una orientación terapéutica, así mismo para conocer el progreso de la enfermedad o para diferenciarla de otra entidad que pueda presentarse conjuntamente. El diagnóstico debe ser sin embargo evolutivo, en la medida que un refuerzo de este a un tiempo prudente ayuda a esclarecer el padecimiento o reorientar el tratamiento. La demencia tipo Alzheimer posee características específicas que lo diferencia de otros tipos de dicha enfermedad, pero el deterioro cognitivo, comportamental y funcional implican caminos más allá de pruebas calificadas para diagnosticarlo, pues ya se vio que le desempeño puede variar por diferentes motivos. El uso de dos o más instrumentos de tamizaje conjunto con un adecuado examen clínico y seguimiento juicioso, permitirán certeza en el diagnóstico y por tanto en el pronóstico y la calidad de vida que pueda obtenerse para el paciente y su familia.

\section{Conclusiones}

La importancia de los estudios sobre la EA innegablemente va más allá del papel de intervención, es decir de la ejecución de programas de psico estimulación, apoyo en unidades geriátricas o bien en servicios de ayuda domiciliaria, la investigación de la enfermedad es de gran trascendencia, mucho más en el país, dónde hay pocos grupos académicos interdisciplinares que se especializan en la profundización de las diversas variables, antecedentes y consecuentes del Alzheimer. Todo resultado que logre realizarse con esta población, cuidadores, familiares y profesionales de la salud, son dignos de ser conocidos para sumar al impacto positivo de un diagnóstico temprano, un tratamiento adecuado o bien proyectos de mejora en su contexto sociocultural y económico.

Cualquier paciente con deterioro cognitivo, debe asegurarse de que su diagnóstico fue dado tras unas pruebas acordes para este fin y con el acceso a las que han demostrado ser sensibles a los puntos de corte para diagnósticos diferenciales. Saber que se tiene una demencia, a que se tiene un tipo Alzheimer cambia todo el panorama de intervención, atención y cuidado por parte de los familiares y profesionales de la salud; permitirá conocer y de alguna manera predecir cuál será el proceso de la enfermedad, las características funcionales más alteradas y en qué periodos de tiempo, lo que facilita el entendimiento y el servicio que se le puede proveer.

\section{Limitaciones del estudio}

Dado que este análisis hace parte de un proyecto de investigación mayor, cuenta con limitaciones como el tamaño de muestra, pues este obedece al grupo final de pacientes voluntarios que contaban con todos los criterios de inclusión, especialmente la aceptación por parte del neuro radiólogo, de ser aptos para el examen de resonancia magnética funcional posterior. Por lo tanto, la muestra se resumió al tamaño óptimo para este tipo de estudios con neuro imágenes. 


\section{Referencias}

1. Peralta L, Ayuso A, Ballesteros Barranco, and A. Rojo Sebastián. Demencias. Medicine. 2019;12(74):4329-4337. h ttps://doi.org/10.1016/j.med.2019.03.011

2. Folstein MF, Folstein SE, McHugh PR. Mini-mental state. A practical method for grading the cognitive state of patients for the clinician. J Psychiatr Res. 1975 nov;12(3):189-198.

3. Nasreddine ZS, Phillips NA, Bédirian V, Charbonneau S, Whitehead V, Collin I, Cummings JL, Chertkow H. The Montreal Cognitive Assessment, MoCA: a brief screening tool for mild cognitive impairment. J Am Geriatr Soc. 2005 abr;53(4):695-699. https://doi.org/10.1111/j.1532-5415.2005.53221.x

4. Fiorenzato E, Weis L, Falup-Pecurariu C, Diaconu S, Siri C, Reali E, Pezzoli G, Bisiacchi P, Antonini A, Biundo R. Montreal Cognitive Assessment (MoCA) and Mini-Mental State Examination (MMSE) performance in progressive supranuclear palsy and multiple system atrophy. J Neural Transm (Vienna) [Internet]. 2016 dic;123(12):1435-1442. https://doi.org/10.1007/s00702-016-1589-3. Epub 2016 Jun 22.

5. Pinto CC, Machado L, Bulgacov T, Rodrigues-Júnior A, Costa M, Ximenes R, Sougey E. Is the Montreal Cognitive Assessment (MoCA) screening superior to the Mini-Mental State Examination (MMSE) in the detection of mild cognitive impairment (MCI) and Alzheimer's Disease (AD) in the elderly? Int. Psychogeriatr [Internet]. 2019; 31(4),491-504. https://doi.org/10.1017/S1041610218001370

6. Markwick A, Zamboni G, de Jager CA. Profiles of cognitive subtest impairment in the Montreal Cognitive Assessment (MoCA) in a research cohort with normal Mini-Mental State Examination (MMSE) scores. J Clin Exp Neuropsychol [Internet]. 2012;34(7):750-757. https://doi.org/10.1080/13803395.2012.672966.

7. Feng C, Xiaoyun XU, Wang Y, Xia GE, Yuanling LI, Jin H, Zhao M. Factors related to the progression of mild cognitive impairment toward Alzheimer's disease. Chin J Phys Med Rehabil. 2016;38(2):108-112. http://doi. org/10.1136/jnnp-2014-310095

8. Benedictus MR, Leeuwis AE, Binnewijzend MA, Kuijer JP, Scheltens P, Barkhof F, van der Flier WM, Prins ND. Lower cerebral blood flow is associated with faster cognitive decline in Alzheimer's disease. Eur Radiol [Internet]. 2017 mar;27(3):1169-1175. https://doi.org/10.1007/s00330-016-4450-z.

9. Cecato JF, Martinelli JE, Izbicki R, Yassuda MS, Aprahamian I. A subtest analysis of the Montreal cognitive assessment (MoCA): which subtests can best discriminate between healthy controls, mild cognitive impairment and Alzheimer's disease? Int Psychogeriatr [Internet]. 2017 abr;29(4):701. https://doi.org/10.1017/S1041610 $21600212 X$

10. Dubois B, Slachevsky A, Litvan I, Pillon B. The FAB: a frontal assessment battery at bedside. Neurology. 2000 dic. 12;55(11):1621-1626. https://doi.org/10.1212/wnl.55.11.1621.

11. Sitges-Maciá E, Bonete-López B. Evaluación del estado cognitivo y afectivo en adultos mayores como estrategia de prevención. Inform Psicol. 2019;117:129-141. https://dx.medra.org/10.14635/IPSIC.2019.117.5

12. Yesavage JA, Brink TL, Rose TL, Lum O, Huang V, Adey M, Leirer VO. Development and validation of a geriatric depression screening scale: a preliminary report. J Psychiatr Res [Internet]. 1982-1983;17(1):37-49. https://do i.org/10.1016/0022-3956(82)90033-4

13. Ekman P, Friesen WV. Constants across cultures in the face and emotion. J Pers Soc Psychol. 1971 feb;17(2):124-129.

14. Rodriguez D, Catalán A, Carrasco M. FAB: a preliminar Spanish application of the frontal assessment battery to 11 groups of patients. Rev Neurol [Internet]. 2003;36(7):605. Disponible en: https://pubmed.ncbi.nlm.nih.g ov/12666037/

15. Reyes D. Tamaño de Muestra Para Considerarse en un Estudio de Resonancia Magnética Funcional (RMF) con un Equipo de Resonancia Magnéticade 1.5 T. Rev. Ecuat. Neurol. 2012; 21(1-3). Disponible en: http://revecuatne urol.com/magazine_issue_article/muestra-a-considerarse-en-estudio-de-resonancia-magnetica-funcional/

16. Kim JW, Lee DY, Seo EH, Sohn BK, Park SY, Choo IH, Youn JC, Jhoo JH, Kim KW, Woo JI. Improvement of dementia screening accuracy of mini-mental state examination by education-adjustment and supplementation of 
frontal assessment battery performance. J Korean Med Sci [Internet]. 2013 oct;28(10):1522-1528. https://doi .org/10.3346/jkms.2013.28.10.1522.

17. De la Vega R, Zambrano A. Deterioro cognitivo leve. La circunvalación del hipocampo [Internet]; 2008 [citado 2019 nov. 22] Disponible en: https://www.hipocampo.org/mci.asp

18. Ferrer MM, Toribio E, Morera G, Sánchez A, Palao S, Gomis JM, Pérez I. Correlación entre la Frontal Assessment Battery (FAB) y diversas escalas de valoración funcional y neurológica en los pacientes estudiados en una unidad de neurología de conducta y demencias. Alzheimer. 2019;41:38-42.

\section{Notas}

* Artículo original de investigación, derivado de la Investigación IMP-HUM 1809. Financiada por la Vicerrectoría de Investigación de la Universidad Militar Nueva Granada, Bogotá, Colombia

\section{Licencia Creative Commons CC BY 4.0}

Cómo citar este artículo: Hackspiel MM, Paredes-Garavito O. Descripción de los resultados de las pruebas neuropsicológicas en el diagnóstico diferencial de los pacientes con Alzheimer. Investig Enferm Imagen Desarr. 2020;22. https://doi.org/10.11144/Javeriana.ie22.drpn 